and ethereal extracts on heating and that, although mercuric acetate lowers the figure (due, according to our observations, not merely to removal of interfering substances but also to a partial loss of ascorbic acid), the increase on heating is significant. In the case of ethereal extracts, the actual figures after heating are low owing to the low solubility of combined ascorbic acid in ether, but it will be observed that before heating the value is nil. Although there are naturally considerable variations in the increase among different cabbage samples, we have observed an increase on heating under our conditions of experiment in all cases without exception.

Further evidence of the presence of combined ascorbic acid in cabbage is provided by experiments (Table II), which show that treatment of cabbage suspensions and extracts with hydrogen sulphide in the hot condition gives a considerably higher value for ascorbic acid than similar treatment at room temperature $\left(25^{\circ} \mathrm{C}\right.$.). Cold treatment with hydrogen sulphide, of course, always gives higher values than the simple method of extraction with trichloroacetic acid, owing to the reduction of the considerable quantities of dehydroascorbic acid present, but the difference between the effects of the hot and cold treatments with hydrogen sulphide indicates again the presence of combined ascorbic acid in cabbage, which is split up by heating. This difference can scarcely be explained on the basis of the oxidase theory, as cold treatment with hydrogen sulphide under our conditions of experiment should be capable of reducing all the dehydroascorbic acid produced by the action of the oxidase.

TABLE 2.

Figures are given in $\mathrm{mgm}$. ascorbic acid per $100 \mathrm{gm}$. material.

\begin{tabular}{|c|c|c|}
\hline \multicolumn{2}{|c|}{ Effect of $\mathrm{H}_{2} \mathrm{~S}$ at $25^{\circ}$} & $\begin{array}{l}\text { Effect of } \mathrm{H}_{2} \mathrm{~S} \text { o: boiling } \\
\text { water bat? }\end{array}$ \\
\hline $\begin{array}{l}\text { Aqueous suspensions } \\
\text { of cabbage }\end{array}$ & $\left\{\begin{array}{l}64 \cdot 5 \\
67 \cdot 0 \\
67 \cdot 8 \\
62 \cdot 5 \\
64 \cdot 0\end{array}\right.$ & $\begin{array}{l}72 \cdot 7 \\
73 \cdot 0 \\
81 \cdot 7 \\
71 \cdot 6 \\
73 \cdot 8\end{array}$ \\
\hline $\begin{array}{l}\text { Aqueous extracts } \\
\text { of cabbage }\end{array}$ & $\begin{array}{l}40 \cdot 0 \\
34 \cdot 6 \\
38 \cdot 5 \\
40 \cdot 0\end{array}$ & $\begin{array}{l}49 \cdot 5 \\
41 \cdot 6 \\
45 \cdot 2 \\
45 \cdot 2\end{array}$ \\
\hline $\begin{array}{l}\text { Alcoholic extracts } \\
\text { of cabbage }\end{array}$ & $\begin{array}{r}12 \cdot 3 \\
9 \cdot 5 \\
21 \cdot 7 \\
24 \cdot 0\end{array}$ & $\begin{array}{l}20 \cdot 3 \\
15 \cdot 0 \\
35 \cdot 3 \\
30 \cdot 0\end{array}$ \\
\hline
\end{tabular}

Further, we have lately observed that chloroform extracts of dried cabbage, which, as such, give no titre with the indophenol reagent, do so after heating in an aqueous suspension, and at least 75 per cent of this reduction value disappears if the extract, after heating, is subjected to the action of a preparation of ascorbic acid oxidase. This is additional evidence for the view that the reducing substance, produced on heating, is very probably ascorbic acid. This view is being further tested by biological experiments.

University College of Science, Calcutta.

B. C. Guha.

Indian Institute for Medical Research, Calcutta.

${ }^{1}$ Guha and Pal, Nature, 137, 946 (1936).

${ }^{2}$ Mack, NATURE, 138, 505 (1936).

${ }^{3}$ Levy, NATURE, 138, 933 (1936).

\section{Magnetic and Electrical Dimensions}

I DouBT very much whether Sir James Henderson ${ }^{1}$ is justified in assuming that the members of the International Committee - and presumably also those of the various congresses and committees which have from time to time reaffirmed the decision that the permeability of space should be regarded as an entity having physical dimensions (the so-called Oslo convention)-were not as fully aware as he himself of "the implications of the adoption of Ampère's theory of magnetism". That their interpretation of these implications differs from his I do not doubt. When I read that "Prof. Howe would have us jettison ... that wonderful machine which has produced the electromagnetic theory of light, to which is due the discovery of the quantum theory and all the other developments of modern theoretical physics", I can only smile and say that it sounds magnificently foolish, but bears little, if any, relation to the facts of the case. In his paper he showed that it was not only possible, but also very tempting, to assume that permeability is merely a numeric, and that in space the magnetic induction $B$ and the magnetizing force $H$ are one and the same thing This is, however, a very old assumption ; Gauss and Weber made it and on it built the electromagnetic system of units, but they never deceived themselves into thinking that it was anything more than a conventional assumption. Sir James is mistaken in thinking that I object to the mathematical nature of his arguments; on the contrary, my objection is to the assumptions which he introduces. The question is : What are the dimensional relations between $H$ and $B$ in space ? I confess in all humility that I do not know, and $\mathrm{I}$ put $B / H=\mu_{0}$, a constant of space analogous to the gravitational or dielectric constants of space. Sir James Henderson, however, says, "According to Ampère's theory $B$ and $H$ are physic. ally identical" and apparently expects the scientific world, which has debated the matter for at least sixty years, to take his word for it.

Prof. W. Cramp asks me what justification I have for writing $\mu \equiv 1 / A^{\prime}$ in preference to $\mu \equiv A^{\prime}$ as suggested by Sir James Henderson. First, Sir James has shown that putting $\mu \equiv A^{\prime}$ is tantamount to assuming that both are numerics, that is to say, by doing this one is making an assumption which prejudges the very question to which an answer is being sought. Secondly, by putting $\mu \equiv A^{\prime}$, the force between two current-carrying coils becomes inversely proportional to the permeability of the medium, which, using $\mu$ in its generally accepted sense, I do not believe. Thirdly, if as Sir James says, " $B$ and $H$ are physically identical" and $\mu \equiv A^{\prime} \equiv$ a numeric, what has become of the property of space to which Prof. Cramp refers? Surely it has been conjured away, and I feel confident that a little detective work will enable Prof. Cramp to discover at what stage of the performance it was made to vanish.
University, Glasgow.
G. W. O. Howe. April 28.

$$
\text { 'NATURE, 139, } 676 \text { (April 17, 1937). }
$$

Is the last paragraph of his letter in NATURE of April 17, Sir James Henderson comes down wholeheartedly on the side of the 'one fluid' theory of magnetism, makes $B$ and $H$ of the same dimensions and, by implication, abolishes $\ell_{0}$. 